\title{
Evaluación de sustratos alternativos en la germinación y crecimiento inicial de Hymenaea courbaril $\mathrm{L}$. en condiciones de vivero
}

\author{
Evaluation of alternative substrates on germination and initial growth of \\ Hymenaea courbaril L. under nursery conditions
}

Alexis Domínguez-Liévano ${ }^{1}$ y Saúl Espinosa-Zaragoza ${ }^{1, *}$

Recibido: 02 marzo 2021 | Aceptado: 20 junio 2021 | Publicado en línea: 30 junio 2021 Citación: Domínguez-Liévano: A; Espinosa-Zaragoza, S. 2021. Evaluación de sustratos alternativos en la germinación y crecimiento inicial de Hymenaea courbaril L. en condiciones de vivero. Revista Forestal del Perú 36(1): 107-117. DOI: http://dx.doi.org/10.21704/rfp.vli36.1707

\begin{abstract}
Resumen
Los productores de plantas en vivero a menudo se enfrentan con diferentes problemas, la principal y no menos importante, es el uso adecuado de sustratos y fertilizantes para obtener plantas con calidad morfológica y fisiológica. El objetivo del presente trabajo de investigación fue evaluar el efecto de diferentes sustratos alternativos sobre el crecimiento inicial en vivero de Hymenaea courbaril L., producidas en bolsas negras de polietileno de $13 \times 20 \mathrm{~cm}$, calibre 500, Volumen $0.98 \mathrm{~L}$. Se utilizó un diseño experimental completo al azar donde los sustratos fueron: tierra negra (testigo) (1), peat moss (1), aserrín de mango + arena de río (2:1), aserrín de mango + peat moss (2:1), tierra negra + peat moss + aserrín de mango (1:1:1) y tierra negra + aserrín de mango (1:1) respectivamente, dando un total de seis tratamientos. Se midió el diámetro del tallo $(\mathrm{mm})$ y altura total $(\mathrm{cm})$. El crecimiento de las plantas se evaluó a lo largo de tres meses a partir de los 14 días posteriores a la siembra. Al finalizar la fase de crecimiento, se realizó la etapa destructiva para obtener los datos: peso seco de parte aérea, peso seco de la raíz, relación parte aérea/raíz, índice de esbeltez e índice de calidad de Dickson. Se encontraron diferencias significativas $(p<0.05)$ entre los tratamientos para las variables altura total, longitud de raíz, peso fresco y diámetro del tallo. Para las variables peso fresco y seco de raíz no se observaron diferencias estadísticas entre los tratamientos. El mejor sustrato para el crecimiento en altura fue; tierra negra (1), para el diámetro de tallo; tierra negra + peat moss + aserrín (mango) (1:1:1) y para la longitud de raíz fue aserrín (mango) + peat moss (2:1).
\end{abstract}

Palabras clave: sustrato, Hymenaea courbaril, calidad de plántula, vivero forestal tradicional, reforestación, programas ambientales

${ }^{1}$ Universidad Autónoma de Chiapas, México.

*Autor de Correspondencia: saulez1@gmail.com 


\begin{abstract}
Nursery plant growers are often faced with different problems, the main and not least of which is the proper use of substrates and fertilizers that will lead to obtain plants with morphological and physiological quality. The objective of the present research work was to evaluate the effect of different alternative substrates on the initial growth in nursery of Hymenaea courbaril L., produced in black polyethylene bags of $13 \times 20 \mathrm{~cm}, 500$ caliber, Volume $0.98 \mathrm{~L}$. A complete randomized experimental design was used where the substrates were: black soil (control) (1), peat moss (1), mango sawdust + river sand (2:1), mango sawdust + peat moss (2:1), black soil + peat moss + mango sawdust (1:1:1) and black soil + mango sawdust (1:1) respectively, giving a total of six treatments. Stem diameter $(\mathrm{mm})$ and total height $(\mathrm{cm})$ were measured. Plant growth was evaluated over three months starting 14 days after planting. At the end of the growth phase, the destructive stage was carried out to obtain the following data: aerial part dry weight, root dry weight, aerial part/root ratio, slenderness index and Dickson's quality index. Significant differences $(p<0.05)$ were found among treatments for the variables total height, root length, fresh weight and stem diameter. For the variables fresh and dry root weight, no statistical differences were observed among treatments. The best substrate for height growth was; black soil (1), for stem diameter; black soil + peat moss + sawdust (mango) (1:1:1) and for root length it was sawdust (mango) + peat moss (2:1).
\end{abstract}

Wey words: substrate, Hymenaea courbaril, seedling quality, traditional forest nursery, reforestation, environmental programs

\section{Introducción}

Los árboles son esenciales en la función de los ecosistemas terrestres, al ayudar a controlar la temperatura, interceptar, adsorber y reflejar la radiación solar. Así pues, el desconocimiento de la importancia de los árboles en la sociedad ha sido una de las causas de deforestación de extensas zonas de vegetación en muchas partes del mundo y en países en vías de desarrollo con importantes recursos forestales. Tala inmoderada, ganadería extensiva, pérdida del suelo, incendios forestales y el uso continuo de agroquímicos, son algunas de las causas de perdida de vegetación (Acero 2000).

Hymenaea courbaril L. se conoce como algarrobo, guapinol, locust, jatobá o courbaril; originario del Trópico Americano (Flores y Benavides 1990, Alzate et al. 2008). En México, se encuentra en las cuatro regiones ecológicas y las 16 formaciones forestales de las 21 que existen; presente en orillas de ríos en los bosques de galerías, tanto en partes altas como en pendientes no muy pronunciadas (MARENA/INAFOR 2002). Además de las propiedades que presenta la madera en la elaboración de muebles y construcción, presenta múltiples propiedades an- tibacteriales, antimicóticas, antiparasitarias y nutricionales. La pulpa de su fruto posee alto contenido de fibra rica en sustancias antioxidantes y con elevada capacidad de absorción de agua (Alzate et al. 2008). Además, Hymenaea courbaril soporta hasta cuatro meses de sequía al crecer en suelos francos y franco-arenosos (Ramos et al. 2002).

Los documentos que se encuentran disponibles sobre la producción de especies forestales tropicales con sustratos a base de aserrín $\mathrm{u}$ otros alternativos locales son limitados (Mateo et al. 2011, 2014; Saldaña 2015; Pinilla et al. 2016; Reyes et al. 2018; Torres et al. 2018a, 2018b; Medina et al. 2020). Por esta razón, la mayoría de las investigaciones realizadas con especies tropicales se sustentan en trabajos evaluados de cultivos de agrícolas.

En la región del Soconusco, Chiapas, actualmente hay información sobre el crecimiento y desarrollo de $H$. courbaril como especie tropical maderable, tanto en bosques naturales, plantaciones o viveros forestales. Por lo que, el objetivo del presente trabajo fue evaluar la respuesta de germinación, crecimiento y desarrollo de plántulas de $H$. courbaril en sustratos 
elaborados con insumos locales en etapa de vivero. Con los resultados obtenidos, se podrá sustituir sustratos comerciales que económicamente tienen un precio alto para el productor. Esto permitiría utilizar sustratos alternativos locales como el aserrín en la producción en vivero, pudiendo reducir el precio de producción de planta, y presentando un potencial de uso en programas de reforestación.

\section{Materiales y Métodos}

El trabajo se realizó en dos etapas: lo primera correspondió a la germinación y desarrollo de las plántulas de $H$. courbaril en un sistema de vivero tradicional; la segunda se llevó a cabo en laboratorio con la fase destructiva. El establecimiento del experimento inicio en el mes de febrero de 2020 y finalizó en el mes de abril de 2020, cuando las plántulas presentaron una altura $\geq 30 \mathrm{~cm}$ y diámetro de tallo $\geq 3.6 \mathrm{~mm}$.

El experimento se llevó a cabo en el vivero forestal del campo experimental de la Universidad Autónoma de Chiapas (UNACH), Facultad de Ciencias Agrícolas, C-IV, en Huehuetán, Chiapas, con coordenadas $15^{\circ} 00^{\prime} 28.4^{\prime \prime}$ latitud norte y $92^{\circ} 24^{\prime} 00.1^{\prime \prime}$ longitud oeste, a una altitud de $20 \mathrm{~m}$. El estudio se realizó en un vivero tradicional con estructura metálica tipo "casa sombra" de $3 \mathrm{~m}$ de alto, cubierta con malla sombra de $70 \%$ luz y $30 \%$ sombra, ventilación lateral y frontal, de color negro y riego manual con regaderas. La temperatura mínima promedio fue de 18 a $23{ }^{\circ} \mathrm{C}$, la media de $24{ }^{\circ} \mathrm{C}$ y la máxima promedio de 26 a $31^{\circ} \mathrm{C}$. El clima es cálido subhúmedo con lluvias en verano Aw0 (w), con precipitación pluvial media anual desde los $1700 \mathrm{~mm}$ hasta más de $3000 \mathrm{~mm}$ (INAFED 2010).

\section{Colecta de frutos y procesamiento de semi- llas}

La colecta de frutos de $H$. courbaril se realizó a finales del mes de enero de 2020 , en un predio particular del municipio de Tapachula de Córdova y Ordoñez, Chiapas. Los árboles se seleccionaron aleatoriamente, considerando buen porte, producción de frutos, sin daños, sin plagas ni enfermedades. Los frutos se trasladaron en bolsas de plástico al laboratorio para luego ser abiertas de manera manual con ayuda de un martillo. La semilla se lavó perfectamente con agua y posteriormente se secaron, quedando en condiciones para la siembra vivero.

\section{Preparación de sustrato}

Se realizó una mezcla de sustratos con tierra negra, peat moss (turba comercial), aserrín de mango (6 meses de descomposición) y arena de río (cribado con malla de $3 \mathrm{~mm}$ de grosor). Las mezclas que se prepararon fueron: tierra negra (testigo) (1), peat moss (1), aserrín de mango + arena de río (2:1), aserrín de mango + peat moss $(2: 1)$, tierra negra + peat moss + aserrín de mango (1:1:1) y tierra negra + aserrín de mango (1:1), dando un total de seis tratamientos (Cuadro 1).

\section{Diseño experimental}

Se utilizó un diseño experimental completamente al azar, se evaluaron seis tratamientos (Cuadro 1) con 20 repeticiones por tratamiento, dando una población total de 120 plantas. Un mes después de la germinación, las plantas estuvieron en la fase de vivero con malla sombra (70\% luz y $30 \%$ sombra), con riegos diarios las primeras tres semanas y posteriormente un riego cada tercer día, hasta completar el tiempo de permanencia en vivero.

\section{Siembra directa}

La siembra fue directa en bolsas negras de polietileno de $13 \times 20 \mathrm{~cm}$, calibre 500 , Volumen $0.98 \mathrm{~L}$ con el sustrato correspondiente; se colocó una semilla por bolsa a una profundidad del doble del tamaño de esta. Como tratamiento pre-germinativo, se aplicó una escarificación física; se colocaron las semillas en agua hirviendo hasta que el agua alcanzo temperatura ambiente y, finalmente se sumergieron las semillas durante $24 \mathrm{~h}$ en agua a temperatura ambiente. Se realizaron conteos diarios durante un mes comenzando 12 días después de la siembra a partir del 01 de febrero al 01 de abril de 2020.

\section{Evaluación de plántulas}

Las mediciones se realizaron en 10 plantas seleccionadas de manera al azar de cada tratamiento; se midió el largo $(\mathrm{cm})$, ancho $(\mathrm{cm}) \mathrm{y}$ 
Evaluación de sustratos en la germinación y crecimiento

\begin{tabular}{cc}
\hline No. de Tratamiento & Descripción \\
\hline A & Tierra negra (testigo) $(1)$ \\
B & Peat moss (1) \\
C & Aserrín de mango + Arena de río $(2: 1)$ \\
D & Aserrín de mango + Peat moss $(2: 1)$ \\
E & Tierra negra + Peat moss + Aserrín de mango (1:1:1) \\
F & Tierra negra + Aserrín de mango (1:1) \\
\hline
\end{tabular}

Cuadro 1. Tratamientos utilizados en vivero para Hymenaea courbaril L.

número de hojas; el diámetro a la base del tallo $(\mathrm{mm})$; la altura total desde la base del tallo hasta la terminación de la yema apical $(\mathrm{cm})$ y; la altura del tallo desde la base del tallo hasta las hojas basales $(\mathrm{cm})$. Las evaluaciones morfológicas en vivero se realizaron cada 14 días a partir de observar las primeras hojas verdaderas. En la fase de laboratorio para llevar a cabo el análisis destructivo, se utilizaron las mismas 10 plantas por tratamiento de la primera etapa de vivero.

\section{Evaluación de variables destructivas}

Finalizado el tiempo de evaluación en vivero, las plantas de cada tratamiento se llevaron al

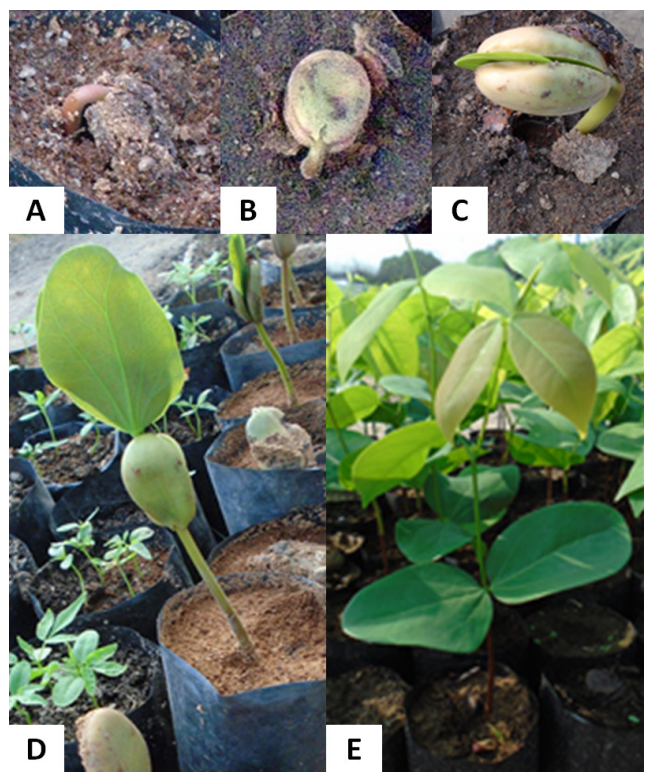

Figura 1. Germinación de Hymenaea courbaril L. (A) Inicio de la germinación. (B) Emergencia de los cotiledones del suelo. (C) Aparición de la primera hoja. (D) Apertura de la hoja. (E) Planta en crecimiento. laboratorio integral de la Facultad de Ciencias Agrícolas para evaluar las variables destructivas. Se retiró el cepellón de cada bolsa para exponer la parte radical, luego se colocaron en recipientes con agua para desprender el excedente de sustrato y poder evaluar. Cada una de las plantas se colocó en bolsas de papel con sus respetivos datos de identificación. Las variables observadas fueron: altura $(\mathrm{cm})$ y diámetro $(\mathrm{mm})$ a la base del tallo. La altura se midió con una regla graduada en centímetros y milímetros; y el diámetro se evaluó con un vernier digital marca Truper $^{\circledast}$ con aproximación a centésimas de $\mathrm{mm}$.

Seguidamente, se separó la hoja, el tallo y raíz de las plantas de cada tratamiento; se pesó la parte aérea y radical con una balanza digital marca OHAUS ${ }^{\circledR}$ con $0.1 \mathrm{~g}$ de precisión. En una estufa marca VWR International TM, modelo $1390 \mathrm{FM}$, se secaron a $90{ }^{\circ} \mathrm{C}$ durante 72 horas. Culminado el tiempo de deshidratación, se pesó la parte aérea y radical para obtener el peso seco. Con los resultados anteriores, se calculó el índice de robustez, índice de lignificación, la relación altura / longitud de raíz, la relación parte aérea / raíz y el índice de calidad de Dickson (ICD) (Dickson et al. 1960), con las siguientes formulas:

$$
\begin{aligned}
& \text { Índice de lignificación }=\frac{\text { Peso seco total }(g)}{\text { Peso fresco total }(g)} \times 100 \\
& \text { Índice de robustez }=\frac{\text { Altura }(\mathrm{cm})}{\text { Diámetro del tallo }(\mathrm{mm})} \\
& \text { Relación parte aérea/raíz }=\frac{\text { Peso seco parte aérea }(g)}{\text { Peso seco de la raíz }(g)} \\
& I C D=\left[\text { Peso seco total }(g) / \frac{\text { Altura }(\mathrm{cm})}{\text { Diámetro }(\mathrm{mm})}+\frac{P_{\text {eso seco aéreo }(\mathrm{g})}}{\text { Peso seco radial }(\mathrm{g})_{2}}\right]
\end{aligned}
$$




\section{Análisis estadístico}

Los datos de las variables obtenidas fueron analizados con el paquete estadístico $\mathrm{R}$ para Windows v3.6.3 (R Core Team 2020); se aplicó el test de normalidad (Shapiro-Wilk) y homogeneidad de varianzas (Bartlett) a los datos, posteriormente se realizó un análisis de varianza (ANOVA) y pruebas de comparación de medias por Duncan, con un nivel de significancia de $p<0.05$.

\section{Resultados}

Las semillas de $H$. courbaril comenzaron a germinar a partir de los doce días después de la siembra, extendiéndose hasta el día 20 (Figura 1). Los resultados de las variables de crecimiento evaluadas en las plántulas de $H$. courbaril presentaron diferencias significativas entre los tratamientos (Cuadro 2).

Los datos obtenidos del análisis estadístico para la altura total, diámetro de tallo y longi- tud de raíz, indicaron una diferencia estadística significativa entre las mezclas de sustratos aplicados. Conforme a los resultados de la prueba de medias por Duncan $(p=0.05)$, el mejor sustrato para el crecimiento en altura fue tierra negra (1) (promedio $=40.67 \mathrm{~cm}$ ), para el diámetro de tallo fue tierra negra + peat moss + aserrín (mango) $(1: 1: 1)$ (promedio $=4.28 \mathrm{~mm})$ y para la longitud de raíz fue aserrín (mango) + peat moss $(2: 1)$ (promedio $=40.98 \mathrm{~cm})$. Para las variables altura total (promedio $=32.03 \mathrm{~cm}$ ) y diámetro del tallo (promedio $=3.70 \mathrm{~mm}$ ) se registraron promedios más bajos en los tratamientos aserrín (mango) + peat moss $(2: 1)$ y peat moss (1), respectivamente. algunos tratamientos; mientras que la tierra negra (testigo) presento el promedio más bajo para la longitud de raíz (promedio $=28.40 \mathrm{~cm}$ ).

En relación con la longitud de raíz, el sustrato testigo (tierra negra) no propició el crecimiento radical $(28.40 \mathrm{~cm})$ debido a las características físicas del mismo; se compactó con los riegos, provocando menos aireación para la raíz,

\begin{tabular}{|c|c|c|c|c|c|c|}
\hline \multirow{2}{*}{ Variables morfológicas } & \multicolumn{6}{|c|}{ Tratamientos } \\
\hline & $\mathbf{A}$ & B & $\mathbf{C}$ & D & $\mathbf{E}$ & $\mathbf{F}$ \\
\hline Diámetro de tallo (mm) & $\begin{array}{l}3.81 \pm \\
0.22^{a b}\end{array}$ & $\begin{array}{l}3.70 \pm \\
0.14^{\mathrm{b}}\end{array}$ & $\begin{array}{l}3.92 \pm \\
0.16^{\mathrm{ab}}\end{array}$ & $\begin{array}{l}4.21 \pm \\
0.14^{\mathrm{ab}}\end{array}$ & $\begin{array}{l}4.28 \pm \\
0.16^{\mathrm{a}}\end{array}$ & $\begin{array}{l}4.26 \pm \\
0.15^{\mathrm{a}}\end{array}$ \\
\hline Altura total $(\mathrm{cm})$ & $\begin{array}{c}40.67 \pm \\
1.77^{\mathrm{a}}\end{array}$ & $\begin{array}{c}36.10 \pm \\
1.23^{\mathrm{ab}}\end{array}$ & $\begin{array}{c}35.53 \pm \\
2.04^{\mathrm{ab}}\end{array}$ & $\begin{array}{c}32.03^{ \pm} \\
1.43^{\mathrm{b}}\end{array}$ & $\begin{array}{c}35.95 \pm \\
1.66^{\mathrm{ab}}\end{array}$ & $\begin{array}{c}40.10 \pm \\
2.22^{\mathrm{a}}\end{array}$ \\
\hline Longitud de raíz (cm) & $\begin{array}{c}28.40 \pm \\
2.56^{\mathrm{b}}\end{array}$ & $\begin{array}{c}35.08 \pm \\
2.91^{\mathrm{ab}}\end{array}$ & $\begin{array}{c}36.21 \pm \\
3.74 \mathrm{ab}\end{array}$ & $\begin{array}{c}40.98 \pm \\
3.65^{\mathrm{a}}\end{array}$ & $\begin{array}{c}38.74 \pm \\
3.60^{\mathrm{a}}\end{array}$ & $\begin{array}{c}36.43 \pm \\
1.71^{\mathrm{ab}}\end{array}$ \\
\hline Peso fresco tallo $(\mathrm{g})$ & $1.75 \pm 0.19^{b}$ & $\begin{array}{l}1.95 \pm \\
0.07^{\mathrm{b}}\end{array}$ & $\begin{array}{l}1.96^{ \pm} \\
0.15^{\mathrm{b}}\end{array}$ & $\begin{array}{l}1.93 \pm \\
0.36^{\mathrm{b}}\end{array}$ & $\begin{array}{l}1.67 \pm \\
0.31^{\mathrm{b}}\end{array}$ & $\begin{array}{l}3.51 \pm \\
0.31^{\mathrm{a}}\end{array}$ \\
\hline Peso seco tallo (g) & $\begin{array}{l}0.76 \pm \\
0.23^{b}\end{array}$ & $\begin{array}{l}0.92 \pm \\
0.10^{\text {ab }}\end{array}$ & $\begin{array}{l}0.96 \pm \\
0.19^{\mathrm{ab}}\end{array}$ & $\begin{array}{l}0.75 \pm \\
0.24^{b}\end{array}$ & $\begin{array}{l}0.81 \pm \\
0.17^{\mathrm{ab}}\end{array}$ & $\begin{array}{l}1.05 \pm \\
0.21^{\mathrm{a}}\end{array}$ \\
\hline Peso fresco hoja $(\mathrm{g})$ & $\begin{array}{l}3.32 \pm \\
0.35^{\mathrm{abc}}\end{array}$ & $\begin{array}{c}3.82 \pm \\
0.15^{\mathrm{a}}\end{array}$ & $\underset{\mathrm{abc}}{3.27 \pm 0.33}$ & $\begin{array}{c}2.46 \pm \\
0.33^{\mathrm{c}}\end{array}$ & $\begin{array}{l}2.88 \pm \\
0.20^{\mathrm{bc}}\end{array}$ & $\begin{array}{l}3.51 \pm \\
0.31^{\mathrm{ab}}\end{array}$ \\
\hline Peso seco hoja (g) & $\begin{array}{l}1.14 \pm \\
0.11^{\mathrm{ab}}\end{array}$ & $\begin{array}{l}1.34 \pm \\
0.05^{\mathrm{a}}\end{array}$ & $\begin{array}{l}1.28 \pm \\
0.11^{\mathrm{a}}\end{array}$ & $\begin{array}{l}0.92 \pm \\
0.12^{\mathrm{b}}\end{array}$ & $\begin{array}{l}1.07 \pm \\
0.06^{\mathrm{ab}}\end{array}$ & $\begin{array}{l}1.33 \pm \\
0.12^{\mathrm{a}}\end{array}$ \\
\hline Peso fresco raíz (g) & $\begin{array}{l}1.89 \pm \\
0.25^{\mathrm{bc}}\end{array}$ & $\begin{array}{c}2.81 \pm \\
0.16^{\mathrm{a}}\end{array}$ & $\begin{array}{l}2.18 \pm \\
0.14^{b}\end{array}$ & $\begin{array}{l}1.61 \pm \\
0.18^{c}\end{array}$ & $\begin{array}{l}1.56 \pm \\
0.15^{\mathrm{c}}\end{array}$ & $\begin{array}{l}1.95 \pm \\
0.13^{\mathrm{bc}}\end{array}$ \\
\hline Peso seco raíz (g) & $\begin{array}{c}0.86 \pm \\
0.13^{a}\end{array}$ & $\begin{array}{l}1.06 \pm \\
0.05^{\mathrm{a}}\end{array}$ & $\begin{array}{l}1.09 \pm \\
0.09^{\mathrm{a}}\end{array}$ & $\begin{array}{c}0.86 \pm \\
0.12^{a}\end{array}$ & $\begin{array}{c}0.81 \pm \\
0.13^{\mathrm{a}}\end{array}$ & $\begin{array}{c}0.99 \pm \\
0.11^{\mathrm{a}}\end{array}$ \\
\hline
\end{tabular}

Cuadro 2. Variables morfológicas obtenidas de los diferentes tratamientos aplicados en plantas de Hymenaea courbaril L. A= Tierra negra (testigo) (1), B= Peat moss (1), C= Aserrín (mango)+Arena de río (2:1), D= Aserrín (mango) + Peat moss (2:1), E= Tierra negra + Peat moss + Aserrín (mango) (1:1:1), F= Tierra negra + Aserrín (mango) (1:1). Las medias seguidas por la misma letra en columna no difieren significativamente entre sí ( $p \leq$ 0.05). Media \pm Error estándar. 
Evaluación de sustratos en la germinación y crecimiento

\begin{tabular}{cccccc}
\hline Tratamientos & $\begin{array}{c}\text { Índice de } \\
\text { robustez }\end{array}$ & $\begin{array}{c}\text { Relación Parte } \\
\text { Aérea / Raíz }\end{array}$ & $\begin{array}{c}\text { Índice de } \\
\text { lignificación }\end{array}$ & $\begin{array}{c}\text { Relación Altura } \\
\text { / Longitud de } \\
\text { Raíz }\end{array}$ & $\begin{array}{c}\text { Índice de } \\
\text { calidad de } \\
\text { Dickson }\end{array}$ \\
\hline $\mathrm{A}$ & $10.83 \pm 0.50^{\mathrm{a}}$ & $1.51 \pm 0.15^{\mathrm{a}}$ & $38.25 \pm 0.90^{\mathrm{bc}}$ & $1.49 \pm 0.09^{\mathrm{a}}$ & $0.16 \pm 0.02^{\mathrm{b}}$ \\
$\mathrm{B}$ & $9.88 \pm 0.52^{\mathrm{ab}}$ & $1.27 \pm 0.04^{\mathrm{ab}}$ & $36.58 \pm 1.65^{\mathrm{c}}$ & $1.15 \pm 0.18^{\mathrm{ab}}$ & $0.21 \pm 0.01^{\mathrm{ab}}$ \\
$\mathrm{C}$ & $9.22 \pm 0.66^{\mathrm{b}}$ & $1.19 \pm 0.06^{\mathrm{ab}}$ & $43.58 \pm 1.38^{\mathrm{a}}$ & $1.08 \pm 0.13^{\mathrm{b}}$ & $0.24 \pm 0.03^{\mathrm{a}}$ \\
$\mathrm{D}$ & $7.65 \pm 0.40^{\mathrm{c}}$ & $1.12 \pm 0.09^{\mathrm{b}}$ & $43.41 \pm 2.47^{\mathrm{a}}$ & $0.82 \pm 0.07^{\mathrm{b}}$ & $0.20 \pm 0.02^{\mathrm{ab}}$ \\
$\mathrm{E}$ & $8.40 \pm 0.31^{\mathrm{bc}}$ & $1.49 \pm 0.16^{\mathrm{a}}$ & $42.01 \pm 1.90^{\mathrm{ab}}$ & $1.00 \pm 0.13^{\mathrm{ab}}$ & $0.19 \pm 0.02^{\mathrm{ab}}$ \\
$\mathrm{F}$ & $9.43 \pm 0.51^{\mathrm{ab}}$ & $1.39 \pm 0.12^{\mathrm{ab}}$ & $42.50 \pm 1.46^{\mathrm{ab}}$ & $1.12 \pm 0.09^{\mathrm{ab}}$ & $0.21 \pm 0.02^{\mathrm{ab}}$ \\
\hline
\end{tabular}

Cuadro 3. Valores de la calidad morfológica de la planta de producida en vivero tradicional de Hymenaea courbaril L. A= Tierra negra (testigo) (1), B= Peat moss (1), C= Aserrín (mango)+ Arena de río (2:1), D= Aserrín (mango) + Peat moss (2:1), E= Tierra negra + Peat moss + Aserrín (mango) (1:1:1), F= Tierra negra + Aserrín (mango) (1:1). Las medias seguidas por la misma letra en columna no difieren significativamente entre sí ( $p \leq$ 0.05). Media \pm Error estándar.

menos espacio poroso y menos infiltración de agua. No obstante, el análisis de varianza arrojo resultados estadísticamente significativos para las variables peso fresco y seco de hojas, peso fresco y seco de la raíz, índice de robustez, relación parte aérea y raíz, índice de esbeltez e índice de calidad de Dickson (ICD) (Cuadro 2 y 3$)$.

En cuanto a los datos obtenidos en la evaluación e interpretación de la calidad de planta (Cuadro 4), H. courbaril se clasificó de calidad alta. Las variables morfológicas evaluadas son; relación parte área / raíz y relación altura / longitud de raíz. Por su parte, las plantas en todos los tratamientos evaluados se clasificaron como calidad media en el índice de robustez e índice de calidad de Dickson.

En la calidad de planta, el mejor tratamiento evaluado desde el punto de vista morfológico fue el aserrín (mango) + arena de río (2:1), con resultados promedios de 9.22 para el índice de robustez, 1.19 en la relación parte aérea / raíz, 43.58 en índice de lignificación, 1.08 relación altura / longitud de raíz y 0.24 en el índice de calidad de Dickson (Cuadro 3). Mientras que, el tratamiento con resultados menores en la calidad morfológica fue el aserrín (mango) + peat moss (2:1) con 7.65 para el índice de robustez, 1.12 en la relación parte aérea / raíz, 43.41 en índice de lignificación, 0.82 relación altura / longitud de raíz y 0.20 en el índice de calidad de Dickson. Para el índice de relación parte aérea y raíz, los mejores tratamientos fueron: A = tierra negra (1) con 1.51 y 1.49 en la mezcla $\mathrm{E}=$ Tierra negra + peat moss + Aserrín (mango) (1:1:1).

\section{Discusión}

En cuanto a los datos obtenidos de germinación, contrastan con los encontrados por Rengifo y Torres (2016), quienes reportan una germinación a partir de los ochos días después de la siembra hasta el día 30. De igual forma, Salas (2014) indicó que el proceso germinativo de $H$. courbaril tarda entre 25 y 30 días, iniciando desde el día 2 y alargándose hasta el día 32. López et al. (2010) y Orozco et al. (2010), concuerdan con que la escarificación mecánica es un tratamiento pre-germinativo efectivo en la estimulación germinativa de las semillas de especies forestales que presentan testa dura como las de H. courbaril.

Rengifo y Torres (2016) mencionan que obtuvieron valores promedios de crecimiento en altura de $27.0 \mathrm{~cm}(p=0.2291)$ con tratamientos pre-germinativos. Señalando de igual forma que, los mejores registros de crecimiento en altura y supervivencia de las plántulas lo obtuvieron en los tratamientos: sin tratamiento pre-germinativo con tierra negra + tierra de hormiga (2:1), sin tratamiento pre-germinativo 


\begin{tabular}{|c|c|c|c|c|}
\hline \multirow{2}{*}{ Características } & \multirow{2}{*}{ Variable } & \multicolumn{3}{|c|}{ Calidad y rango } \\
\hline & & Alta & Media & Baja \\
\hline \multirow{5}{*}{ Morfológicas } & Altura $(\mathrm{cm})$ & $\geq 12.0$ & $10.0-11.9$ & $<10.0$ \\
\hline & Diámetro (mm) & $\geq 5.0$ & $2.5-4.9$ & $<2.5$ \\
\hline & Índice de robustez & $<6.0$ & $7.9-6.0$ & $\geq 8.0$ \\
\hline & Relación altura: longitud de raíz & $\leq 2$ & $2.1-2.5$ & $>2.5$ \\
\hline & $\begin{array}{c}\text { Relación biomasa seca aérea/biomasa } \\
\text { seca raíz }\end{array}$ & $1.5-2.0$ & $2.1-2.5$ & $>2.5$ \\
\hline Fisiológica & Índice de calidad de Dickson & $\geq 0.50$ & $0.49-0.20$ & $<0.20$ \\
\hline
\end{tabular}

Cuadro 4. Valores utilizados en especies latifoliadas como referencia para determinar la calidad de planta de Hymenaea courbaril L. en vivero. Fuente: Elaborado con base en Muñoz et al. 2014, Sáenz et al. 2010, Santiago et al. 2007 y CONAFOR 2009.

con arena aluvial y escarificación mecánica con arena aluvial, con valores de $31(\mathrm{p}=0.2630)$, $30.2(p=0.2562)$ y $30.0 \mathrm{~cm}(p=0.2545)$, respectivamente. Además, Salas (2014) indicó que en cuatro meses obtuvo resultados de crecimiento en altura para $H$. courbaril de 25.8 y $27.5 \mathrm{~cm}$, valores similares a los encontrados por Rengifo y Torres (2016) en el mismo tiempo de evaluación.

Flores et al. (2015) evaluaron el efecto de tratamientos pre-germinativos para conocer la calidad de planta de $H$. courbaril, mostrando que el mayor diámetro $(3.33 \mathrm{~mm})$ y la mayor altura $(18.58 \mathrm{~cm})$ se presentó en las semillas con hidratación, y el menor diámetro $(2.43 \mathrm{~mm})$ en el tratamiento testigo. Señalan de igual manera, que las plantas crecieron menos en altura cuando compararon el tratamiento químico (14.60 $\mathrm{cm})$ con el tratamiento térmico $(15.40 \mathrm{~cm})$ y el tratamiento mecánico $(17.60 \mathrm{~cm})$.

Por lo anterior, con los tratamientos evaluados en la presente investigación se consiguieron mejores resultados en altura y diámetro. Asimismo, nuestros resultados fueron mayores en comparación con los reportados por Orozco et al. (2010) para algunas especies tropicales producidas en viveros del estado de Colima (México) con sustratos alternativos similares; alturas promedio de $16.3 \mathrm{~cm}$ (Caesalpinia platyloba S. Watson), $18.9 \mathrm{~cm}$ (Swietenia humilis Zucc) y $22.5 \mathrm{~cm}$ (Tabebuia donnell-smithii Rose).
De acuerdo con Reyes et al. (2005), se pueden conseguir resultados favorables al utilizar sustratos alternativos como el aserrín en la producción de plantas en viveros tecnificados. Para Pinus pseudostrobus var. apulcensis (Lindl.) Shaw registraron un mayor diámetro $(3.33 \mathrm{~mm})$ y altura $(18.58 \mathrm{~cm})$ en plántulas desarrolladas en sustratos compuestos por $80 \%$ aserrín $+20 \%$ peat moss. No obstante, el menor diámetro $(2.43 \mathrm{~mm})$ se dio en la mezcla de $80 \%$ aserrín $+20 \%$ tierra de monte, sin encontrar diferencias estadísticamente significativas en comparación con la mezcla compuesta por $80 \%$ aserrín $+20 \%$ corteza de pino $(2.46 \mathrm{~mm})$. Observaron de igual forma, un menor crecimiento en altura $(14.60 \mathrm{~cm})$ con el tratamiento constituido de $80 \%$ aserrín $+20 \%$ corteza de pino, aunque resultara estadísticamente igual a las mezclas de $80 \%$ aserrín $+20 \%$ tierra de monte $(15.40 \mathrm{~cm})$ y de $80 \%$ aserrín $+20 \%$ agrolita $(17.60 \mathrm{~cm})$. Por lo que, hay un mejor crecimiento de las plántulas con el sustrato a base de aserrín con peat moss.

Así mismo, los resultados de Reyes et al. (2005), de manera general, coinciden con lo realizado por Mateo (2002) en las especies de Pinus patula Schiede ex Schltdl. \& Cham. y Pinus teocote Schied. ex Schltdl. \& Cham., encontrando que, si las mezclas contenían entre 70 y $80 \%$ de aserrín con fertilizante se produce mayor peso seco, altura y diámetro en las plantas de ambas especies al hacer una comparación de 
Evaluación de sustratos en la germinación y crecimiento

mezclas de 10 a 100\% de aserrín con tierra de monte.

Respecto al peso fresco y seco del tallo, el análisis de varianza reflejó diferencias significativas entre los tratamientos (Cuadro 2). Los valores obtenidos de la prueba de medias por Duncan ( $p=0.05)$, arrojaron que el tratamiento que presento mejor respuesta a las variables antes mencionadas fue tierra negra + aserrín (mango) (1:1), a diferencia de aserrín (mango) + peat moss $(2: 1)$ y tierra negra + peat moss + aserrín (mango) (1:1:1), en el que se observaron menor peso fresco y seco del tallo respectivamente.

En cuanto al diámetro de tallo, la mezcla de tierra negra + peat moss + aserrín (mango) (1:1:1), de acuerdo al análisis de varianza, alcanzaron los mejores resultados a diferencia de otros tratamientos. El diámetro de tallo es un indicador importante ya que está relacionada con la vigorosidad de la planta, no solo por la información que brinda en sí, sino porque permite conocer el porte general del movimiento de nutrimentos que va a llegar a tener la planta (Quesada y Méndez 2005). Entre los tratamientos, el peat moss (1) fue el que registró el menor incremento en el diámetro de tallo $(3.70 \mathrm{~mm})$, debido a las características del sustrato que no permitieron el adecuado crecimiento del tallo (turba solida e inerte) (Borges 1998, Abad et al. 1999).

Los tratamientos que registraron menor crecimientos en altura, diámetro de tallo y longitud de raíz fueron; peat moss (1), aserrín (mango) + peat moss $(2: 1)$ y tierra negra (1), respectivamente. El bajo crecimiento que presentaron las plantas de $H$. courbaril se le atribuye al tipo de sustrato utilizado; el aserrín como sustrato alternativo contiene altos valores de $\mathrm{C} / \mathrm{N}$, según el grado de descomposición. Además, la acción de microorganismos que interactúan con el aserrín en su descomposición no es favorable en el desarrollo de las plantas, ya que no permiten la adsorción de nutrimentos disponibles que pudieran estar presentes en la combinación de otros sustratos.

Con el peat moss sucedió algo similar al no ser una fuente de nutrimentos, a diferencia de la tierra negra que si aporta nutrientes disponibles para las plantas. Sin embargo, el inconveniente que presenta la tierra negra, en el crecimiento en longitud de la raíz, es su textura porque este sustrato se compacta con los riegos y no permite el desarrollo de las raíces.

Los resultados obtenidos, demuestran que el tipo de sustrato y la mezcla utilizada influyó positivamente según el valor conseguido en la altura de la planta, diámetro del tallo y longitud de raíz, como principales variables observadas. En el Cuadro 3, se presentan los valores medios derivados de calidad morfológica de la planta producida en el vivero.

No obstante, se tendrá que correlacionar variables como la altura con otros criterios agroclimáticos para determinar la calidad de planta y sus dimensiones futuras en campo en relación con la supervivencia. Por lo anterior, la altura es importante en la observación del desarrollo y comportamiento de las plantas en campo, pero no resulta como un indicador adecuado cuando las condiciones de sitio son desfavorables (hierba, arbustos y tipos de suelo) (Mexal y Landis 1990). Así mismo, Mexal y Landis (1990) mencionan que el diámetro de tallo se encuentra dentro de las características importantes en la predicción de la supervivencia de las plantas en campo, por lo que, definir la robustez del tallo es fundamental. Así pues, dentro de las ventajas de presentar un diámetro mayor $(>5 \mathrm{~mm})$ se encuentran: resistencia al doblamiento por el viento, tolerancia a daños por plagas o daños mecánicos y por las acciones de los animales presentes en la zona de plantación.

De acuerdo con los resultados de relación parte aérea / raíz, la mejor planta será considerada aquella que tenga el mejor resultado ( $\leq$ 2), ya que la distribución de la parte aérea y la longitud radical se encontrará en equilibrio, garantizando una mayor probabilidad de supervivencia (May 1984, Thompson 1985).

En el índice de robustez se encontraron diferencias estadísticas $(p=0.05)$ respecto al análisis de comparación de medias. Para este índice, Thompson (1985) recomienda que el valor 
deberá estar debajo de 6 para tener una mejor asociación entre una planta robusta con un tallo vigoroso. El índice de robustez más cercano al recomendado por Thompson (1985), fue obtenido con el tratamiento $\mathrm{D}=$ aserrín (mango) + peat moss (2:1), con un valor de 7.65. Asimismo, el valor más alejado (10.83) se obtuvo con el tratamiento $\mathrm{A}=$ tierra negra (testigo) (1).

Por último, en el índice de calidad de Dickson se obtuvo una diferencia estadística significativa $(p=0.05)$ entre tratamientos. El tratamiento $\mathrm{C}=$ Aserrín (mango)+ Arena de río (2:1), tuvo un mejor resultado (0.24), por su parte el tratamiento $\mathrm{A}=$ Tierra negra (1), fue el más bajo (0.16). Lo que determina este índice en general es el grado de resistencia de las plantas a factores agroambientales desfavorables que se van a estar expresando en el sitio de plantación (Ritchie 1984).

\section{Consideraciones Finales}

El desarrollo y crecimiento de las plantas de $H$. courbaril dependió del sustrato que se utilizó y las mezclas que se elaboraron, se observó diferencias estadísticas entre los tratamientos para altura total, diámetro de tallo y longitud de raíz. El tratamiento que presento mejores resultados para la altura total fue el de tierra negra + aserrín (mango) (1:1), para la longitud de raíz fue el de aserrín (mango) + peat moss (2:1) y para el diámetro de tallo fue el de tierra negra + peat moss + Aserrín (mango) (1:1:1).

Los tratamientos que contenían sustratos mezclados con aserrín de mango de seis meses de descomposición presentaron resultados favorables en las variables morfológicas: altura total, diámetro de tallo y longitud de raíz. Sin embargo, tal acción no sucedió en mezclas con condiciones físicas o de estructura semejante al del aserrín, tal es el caso del tratamiento con peat moss. Teniendo en consideración que las plantas fueron producidas en condiciones de vivero tradicional, los resultados aportan una alternativa a la producción de $H$. courbaril para fines de reforestación y que además es económicamente rentable. En conclusión, los resultados obtenidos son prácticos y podrán ayudar en la producción de plantas de $H$. courbaril cuando los recursos económicos no sean suficientes. Por lo tanto, es factible la utilización del aserrín de mango como sustrato alternativo en la producción de plantas de $H$. courbaril en vivero tradicional.

\section{Referencias}

Abad, BM; Noguera, P; Noguera, V; Segura, ML. 1999. Los sustratos para el semillero hortícola. In Vilarnau, A; González, J (eds.). Planteles, semilleros y viveros. Tarragona, España, Ediciones de Horticultura. p. 56-58.

Acero, AT. 2000. Flora medicinal empleada en el tratamiento de enfermedades respiratorias y gastrointestinales en dos comunidades zoques de Chiapas. Tesis Lic. Chiapas, México, UNICACH. 94 p.

Alzate, LM; Arteaga, DM; Jaramillo, Y. 2008. Propiedades farmacológicas del algarrobo $(\mathrm{Hy}$ menaea courbaril Linneaus) de interés para la industria de alimentos. Revista Lasallista de Investigación 5 (2):100-111.

Borges, GL. 1998. Uso de sustratos regionales en la agricultura yucateca. Revista de la Academia Mexicana de Ciencias 49(1):21-26.

CONAFOR (Comisión Nacional Forestal). 2009. Paquetes tecnológicos. Criterios técnicos para la producción de especies forestales de ciclo corto con fines de restauración. Taxco, Gro. México. 9 p.

Dickson, A; Leaf, AL; Hosner, JF. 1960. Quality appraisal of write spruce and white pine seedling stock in nurseries. The Forestry Chronicle 36(1):10-13.

Flores, PJA; Ramírez-James, M; Gutiérrez-Rugama, A; Flores-Pacheco, CJ; Alemán, Y. 2015. Efecto de tratamientos pre-germinativos en la calidad de plántulas guapinol (Hymenaea courbaril). Revista Científica Nexo 28(2):8396.

Flores, ME; Benavides, CE. 1990. Germinación y morfología de la plántula de Hymenaea courbaril L. (Caesalpinaceae). Revista de Biología Tropical 38(1):91-98. 
Evaluación de sustratos en la germinación y crecimiento

INAFED (Instituto para el Federalismo y el Desarrollo Municipal, México). 2010. Huehuetán. In Enciclopedia de los municipios y delegaciones de México. Ciudad de México, México. v. 1, p. 2-3.

López, D; Hernández, JA; Rodríguez, PB; Orantes, C; Garrido, ER. 2010. Efecto de la escarificación mecánica e inmersión en agua caliente, sobre el letargo de semillas de guapinol (Hymenaea courbaril L. (Fabaceae)). Lacandonia 4(2):37-51.

MARENA (Ministerio del Ambiente y los Recursos Naturales, Nicaragua) / INAFOR (Instituto Nacional Forestal, Nicaragua). 2002. Guía de especies forestales de Nicaragua. Managua, Nicaragua. 304 p.

Mateo, SJJ. 2002. Potencial del aserrín como alimento para rumiantes y sustrato para plantas. Tesis PhD. Montecillo, México, Colegio de Postgraduados. México. 92 p.

Mateo, SJJ; Bonifacio, VR; Pérez, SR; Mohedano, CL; Capulín, GJ. 2011. Producción de Cedrela odorata L. en sustrato a base de aserrín crudo en sistema tecnificado en Técpan de Galeana, Guerrero, México. Ra Ximhai (7):123-132.

Mateo, SJJ; Capulín-Grande, J; Araujo-Santana, MR; Suárez-Islas, A; Mitjans-Moreno, B. 2014. Crecimiento de Acacia retinodes Schltdl. en sustratos a base de aserrín de pino y envases tratados con cobre. Revista Cubana de Ciencias Forestales 2(2):16.

May, JT. 1984. Seedling quality, grading, culling and counting. In May, JT; Belcher, W; Cordell, CE; Filer, TH; South, D; Lantz, CW (eds.). Southern pine nursery handbook. Georgia, Estados Unidos de América, USDA. p. 83-97.

Medina, AHH; Torres-Torres, JJ; Palacios-Palacios, CA; Ruiz-Blandón, BA; Martínez-Guardia, M; Rengifo-Murillo, L. 2020. Germination and growth of the tree Handroanthus chrysanthus (Bignoniaceae) under nursery conditions. UNED Research Journal 12(2): e3175.

Mexal, J; Landis, T. 1990. Target seedling concepts: height and diameter. In Rose, R; Campbell, SJ; Landis, TD (eds.). Target seedling symposium: proceedings of the combined meeting of the western forest nursery associations. Oregon, Estados Unidos de América, USDA. p. 17-34.

Muñoz, FHJ; Sáenz-Reyes, JT; Coria-Avalos, VM; García-Magaña, JJ; Hernández-Ramos, J; Manzanilla-Quijada, GE. 2015. Calidad de planta en el vivero foestal La Dieta, Municipio Zitácuro, Michoacán. Revista Mexicana de Ciencias Forestales 6(27):72-89.

Orozco, G; Muñoz, HJ; Rueda, A; Sígala, JÁ; Prieto, JÁ; García, JJ. 2010. Diagnóstico de la calidad de planta en los viveros forestales del estado de Colima. Revista Mexicana de Ciencias Forestales (Nota técnica) 1(2):134-145.

Pinilla, CH; Medina, AHH; Córdoba, UC; Torres, TJJ; Córdoba, MJC; Mosquera, AY; Martínez, GM. 2016. Propagación y crecimiento inicial del abarco (Cariniana pyriformis Miers), utilizando semillas silvestres. Revista de Investigación Agraria y Ambiental 7(2):87-97.

Quesada, RG; Méndez, SC. 2005. Evaluación de sustratos para almácigos de hortalizas. Agronomía Mesoamericana 16(2):171-183.

R Core Team. 2020. R: a language and environment for statistical computing. Vienna, Austria, R Foundation for Statistical Computing.

Ramos, YA; García, F; Perea, M; Parra, A; Córdoba, H; Sánchez, N; Córdoba, JA; Serna, D. 2002. El algarrobo (Hymenaea courbaril L.) y el castaño (Compsonneura atopa (A.C. Sm.): dos especies alimenticias del departamento del Chocó en peligro de extinción. Revista Institucional Universidad Tecnológica del Chocó (15):72-77.

Rengifo, L; Torres, JJ. 2016. Manejo en vivero de Hymenaea courbaril L. (Fabaceae) en el municipio de Unión Panamericana, Chocó, Colombia. Investigación, Biodiversidad y Desarrollo 35(1):11-9.

Reyes, RJ; Aldrete, A; Cetina-Alcalá, VM; López-Upton, J. 2005. Producción de plántulas de Pinus pseudostrobus var. apulcensis en sustratos a base de aserrín. Revista Chapingo (Serie Ciencias Forestales y del Ambiente) 11(2):105-110. 
Reyes, RJ.; Pimienta, DJ; Rodríguez-Morales, JA; Fuentes-Pérez, MA; Palomeque-Figueroa, E. 2018. Calidad de planta de Gmelina arborea Roxb. producida con diferentes mezclas de sustratos en vivero. Revista Mexicana de Ciencias Forestales 9(47):111-130.

Ritchie, GA. 1984. Assessing seedling quality. In Duryea, ML; Landis, TD (eds.) Forestry nursery manual: production of bareroot seedlings. Oregon, Estados Unidos de América, Oregon State University. 65 p.

Sáenz, RJT; Muñoz, FHJ; Villaseñor, RF; Prieto, RJA; Rueda, SA. 2010. Calidad de planta en viveros forestales de clima templado en $\mathrm{Mi}$ choacán. Folleto Técnico Núm. 12. SAGARPA-INIFAP-CIRPAC-Campo Experimental Uruapan. Uruapan, Mich. México. 50 p.

Salas, AJ. 2014. Efectos de agentes proxidantes y antioxidantes sobre la germinación de las semillas de Hymenea oblingifolia Huber. "Azúcar huayo". Tesis Lic. Puerto Maldonado, Perú, UNAMAD. 38 p.

Saldaña, GLP. 2015. Crecimiento y sobrevivencia, en vivero, de plántulas de Cedrelinga catenaeformis "tornillo", en diferentes sustratos. Tesis Lic. Iquitos, Perú, UNAP. 72 p.

Santiago, OT; Sánchez, V; Monroy, MR; García, S. 2007. Manual de producción de especies forestales tropicales en contenedor. Veracruz, México, INIFAP. 73 p. (Folleto técnico n. 44).

Thompson, B. 1985. Seedling morphological evaluation- what you can tell by looking. In Duryea, ML (ed.). Proceedings: evaluation seedling quality: principles, procedures, and predictive abilities of mayor test. Oregon State University, Estados Unidos de América. p. 5971.

Torres, TJJ; Medina, AJJ; Martínez, GM. 2018a. Germinación y crecimiento inicial de Cedrela odorata L. (Sapindales: Meliaceae), empleando semillas silvestres en el departamento del Chocó, Colombia. Revista Biodiversidad Neotrópica 8(1):22-8.

Torres, TJJ; Medina, AHH; Martínez, GM. 2018b. Germinación de semillas silvestres de
Apeiba glabra Aubl. (Malvaceae) y crecimiento inicial de plantas. Corpoica Ciencia y Tecnología Agropecuaria 19(2):337-349. 